\title{
Editorial
}

\section{Ranking de universidades latinoamericanas 2017}

Los rankings universitarios, más allá de constituir un fenómeno reciente derivado de la globalización, como refieren Marope, Wells y Hazelkorn (2013), es una señal inequívoca de que las universidades viven realmente "en una época de mediciones y comparaciones". Desde principios del siglo XXI, de acuerdo con Marginson y Van der Wende (2007), la competencia entre las universidades se ha intensificado en diferentes ámbitos de la gestión universitaria, cubriendo aspectos como la obtención de mayores fondos y recursos financieros, la competencia por los estudiantes y por los mejores profesores $y$, desde comienzos del 2000 la competencia por el prestigio institucional medido a través de la posición obtenida por las universidades en los rankings más reconocidos a nivel internacional.

Los comparativos mundiales entre las universidades, si bien es cierto tienden a favorecer las actividades de investigación sobre la docencia, también pueden producir cambios favorables en las prácticas de enseñanza aprendizaje (Institute for Higher Education Policy, 2009), por ejemplo, al implementar cursos que son dictados en idiomas extranjeros o al impulsar una política integral de internacionalización académica. El cotejo de las universidades entre sí constituye algo positivo si las conduce a la posibilidad de replicar programas que ya tuvieron éxito en otros lugares, concediendo, a través de la comparación institucional, la identificación y la adopción de mejores prácticas académicas y organizacionales (Villaseñor Moreno y Flores, 2015).

Los rankings vistos así, pasan a convertirse en instrumentos de motivación de cambio en las universidades; a decir de Baty (2012), en herramientas que facilitan la cooperación entre ellas y la atracción de talento e incluso, de acuerdo con el Institute for Higher Education Policy (2009), "fomentan la colaboración, así como las asociaciones para la investigación, programas de movilidad para estudiantes y profesores [...] y pueden ser importantes puntos de partida para identificar a las instituciones con las cuales colaborar y asociarse".

Entre los rankings internacionales referentes se puede citar al Academic Ranking of World Universities (ARWU) de la Universidad Jiao Tong de Shanghái, China; Times Higher Education World University Rankings (THE); Webometrics Ranking of World Universities, y el QS World University Rankings (QS). Estos utilizan indicadores y metodologías diversas y tienen niveles diferenciados de credibilidad e impacto. También existen otros como el Scimago Institutions Rankings (SIR) y el del Centre for Science and Technology Studies (CWTS) de la Universidad de Leiden, reconocidos entre especialistas de educación superior, pero con menos presencia en medios de comunicación.

Para quienes cuestionan los resultados de los rankings, el sesgo que tienen estas herramientas no sería favorable para la mayoría de las universidades. Este sesgo, según ellos, se expresa en lo siguiente: casi todas se concentran en la medición de indicadores asociados a la circulación internacional de la producción de investigación como número de premios Nobel, artículos en revistas indizadas en Web of Science o Scopus (que favorece más a los académicos y universidades de habla inglesa y difundir sobre todo contenidos de áreas biológicas y de la salud), académicos altamente citados ( $\mathrm{HiCi}$ ), artículos en Nature y Science y citas por artículo, entre otros (Ordorika, 2015). Las actividades de formación de estudiantes, de extensión universitaria y difusión de la cultura, y la atención a diversas responsabilidades y compromisos con la sociedad, todas ellas funciones sustantivas de las universidades, están prácticamente ausentes de los rankings (Ordorika, 2015).

De acuerdo con el Ranking QS, la ubicación de las mejores universidades para Latinoamérica al 2017 son: 1) Universidade de Sao Paulo, 2) Universidade Estadual de Campinas (Unicamp), 3) Pontificia Universidad Católica de Chile (UC), 4) Universidad Nacional Autónoma de México, 5) Universidade Federal do Rio de Janeiro, 6) Universidad de Chile, 7) Instituto Tecnológico y de Estudios Superiores de Monterrey (México), 8) Universidad de los Andes (Colombia), 9) Universidade de Brasilia y 10) Universidad Nacional de Colombia. La metodología de este ranking está basada en la reputación académica de la universidad, reputación del empleador, estudiantes, personal con doctorado, impacto de la web, artículos científicos, citaciones por artículo, y red internacional de investigación (QS University Rankings, 2017).

The Times Higher Education World University Rankings (THE) para el 2017 determinó el siguiente orden 1) Universidade Estadual de Campinas, 2) Universidade de São Paulo, 3) Pontificia Universidad Católica 
de Chile, 4) Universidad de Chile, 5) Universidad de los Andes (Colombia), 6) Instituto Tecnológico y de Estudios Superiores de Monterrey (México), 7) Universidad Federal de Sao Paulo, 8) Universidade Federal do Rio de Janeiro, 9) Pontificia Universidad Católica de Rio de Janeiro y 10) Universidad Nacional Autónoma de México. La metodología empleada por el Ranking THE está basada en mediciones de las dimensiones enseñanza, investigación, citaciones de las publicaciones, perspectiva internacional e ingresos por transferencia de conocimiento (The World University Rankings, 2017).

En ambos rankings latinoamericanos también se puede evidenciar las diferencias respecto a la metodología, y un aspecto que resalta es la presencia de la dimensión de investigación componente que además sucede en los rankings regionales y nacionales de diversos países.

Para muchos tomadores de decisiones los rankings no son herramientas útiles de evaluación de la calidad de las instituciones de educación superior (Ordorika, 2015), sobre todo porque existen diversos modelos de universidades con diferentes visiones que no necesariamente se ajustan a los múltiples y sesgados criterios de medición internacional; pero, es una realidad, estos están presentes y muchas personas los consideran con variados fines; toda una tendencia a la cual tenemos que acostumbrarnos.

\section{Referencias bibliográficas}

Institute for Higher Education Policy. (01 de 01 de 2009). Impact of College Rankings on Institutional Decision Making: Four Country Case Studies. Washington DC, USA. Obtenido de http:// www.ihep.org/publications/publications-detail. $\mathrm{cfm}$ ? $\mathrm{id}=126$
Marginson, S., \& Van der Wende, M. (2007). To Rank or To Be Ranked: The Impact of Global Rankings in Higher Education. Journal of Studies in International Education, 306-329. doi: $10.1177 / 1028315307303544$

Marope , P., Wells, P., \& Hazelkorn, E. (2013). Rankings and accountability in Higher Education: uses and misuses. Paris: UNESCO Publishing.

Ordorika, I. (2015). Rankings universitarios. Revista de la Educación Superior, Vol. XLIV(173), 7-9.

QS University Top Universities. (2017). QS University Rankings Latin America. Obtenido de https:// www.topuniversities.com/university-rankings/latinamerican-university-rankings/2016

The World University Rankings. (2017). Latin America University Rankings 2017. Obtenido de https:// www.timeshighereducation.com/world-universityrankings/2017/latin-america-universityrankings\#!/page/0/length/25/sort_by/rank/sort_ order/asc/cols/stats

Villaseñor Becerra, J., Moreno Arellano, C., \& Flores Orozco, J. (2015). Perspectivas actuales sobre los rankings mundiales de universidades. Revista de la Educación Superior, 175, 41-67.

Wilfredo Bulege Gutiérrez

Revista Apuntes de Ciencia \& Sociedad

Editor en Jefe

wbulege@continental.edu.pe 